\title{
The integrated satellite-acoustic telemetry (iSAT) system for tracking marine megafauna
}

\author{
Pedro R. De La Torre ${ }^{1}$, Khaled N. Salama ${ }^{1}$, Michael L. Berumen ${ }^{1,2}$, E. Lloyd Smith ${ }^{1}$ \\ ${ }^{1}$ King Abdullah University of Science and Technology, Thuwal, 23955-6900 Saudi Arabia \\ ${ }^{2}$ Woods Hole Oceanographic Institution, Woods Hole, MA 02543 USA \\ e-mail: \{pedro.torre, khaled.salama, michael.berumen, lloyd.smith\}@kaust.edu.sa
}

\begin{abstract}
This document describes the integrated satelliteacoustic telemetry (iSAT) system: an autonomous modular system for tracking the movements of large pelagic fish using acoustic telemetry and satellite communications. The sensor platform is described along with the propulsion and navigation systems. An application for tracking the whale shark (Rhincodon typus) in the Red Sea is included along with a discussion of the technical difficulties that such a system faces.
\end{abstract} typus

Keywords- satellite tracking; acoustic telemetry; Rhincodon

\section{INTRODUCTION}

Monitoring the behavior of marine species requires specialized tools that can cope with the harsh marine environment and potentially extreme conditions present at sea. Modern commercial technology available to study the movements of fish is divided into three principal categories: acoustic telemetry, satellite tracking, and light-based geolocation. Acoustic telemetry provides high spatial resolution but is also spatially limited by the extent of the network of receivers that need to be installed in a study area. Also, the sea state: wave motion, currents etc, and the physical characteristics of the monitored area [1] along with absorption by suspended particles in the water, signal destructive interference and the motion of the tagged species behind or around obstacles affect the effective reception of signals generated by acoustic tags [2]. However, it is a useful tool to describe site fidelity and patterns of motion of fish in ecosystems like coral reefs [3]. Satellite tracking equipment, mainly for pelagic fish and long distances swimmers like tunas or sharks, provides global coverage of movement but generally sacrifices spatial resolution. Another limitation of satellite tags is the frequent failure to effectively transmit collected data due to one or more of several potential problems. These include but are not limited to tag structural failure due to excessive mechanical stress, satellite unavailability for transmission of information, a tag that is never exposed to air and therefore can never broadcast to a satellite, or tag loss due to retrieval difficulty: inaccessible areas, both geographically and politically, very long traveling distances to the tag and others that make retrieval logistically complex [4].

Finally, light-based systems for detection of position are a useful tool for long range, in the order of hundreds of kilometers, low accuracy, in the order of latitudinal degrees [5], measurements of movements. Hence, they are not a suitable instrument to be used in species like, for example, coral reef fish with smaller spatial ranges. Furthermore, tag retrieval is necessary along with the problems mentioned before, and data post processing that requires specialized filters and adjustments.

In this document a technology under development that combines the advantages of acoustic underwater telemetry together with the benefits of satellite tracking is described. The integrated satellite-acoustic telemetry (iSAT) system is a new tool designed to unveil the movement patterns of marine species with high spatial resolution while maintaining global coverage. In general terms, iSAT is a vessel that surfs the sea equipped with an array of hydrophones submerged under the keel that constantly monitor a target signal generated from an electronic tag attached to a species whose movements are being studied. Also, iSAT has numerous telecommunication networks at its disposal to maximize robustness and availability of communications. The iSAT instruments are mounted on a platform where a variety of additional marine and atmospheric sensors can be deployed, providing further in-situ measurements of the environmental conditions surrounding the

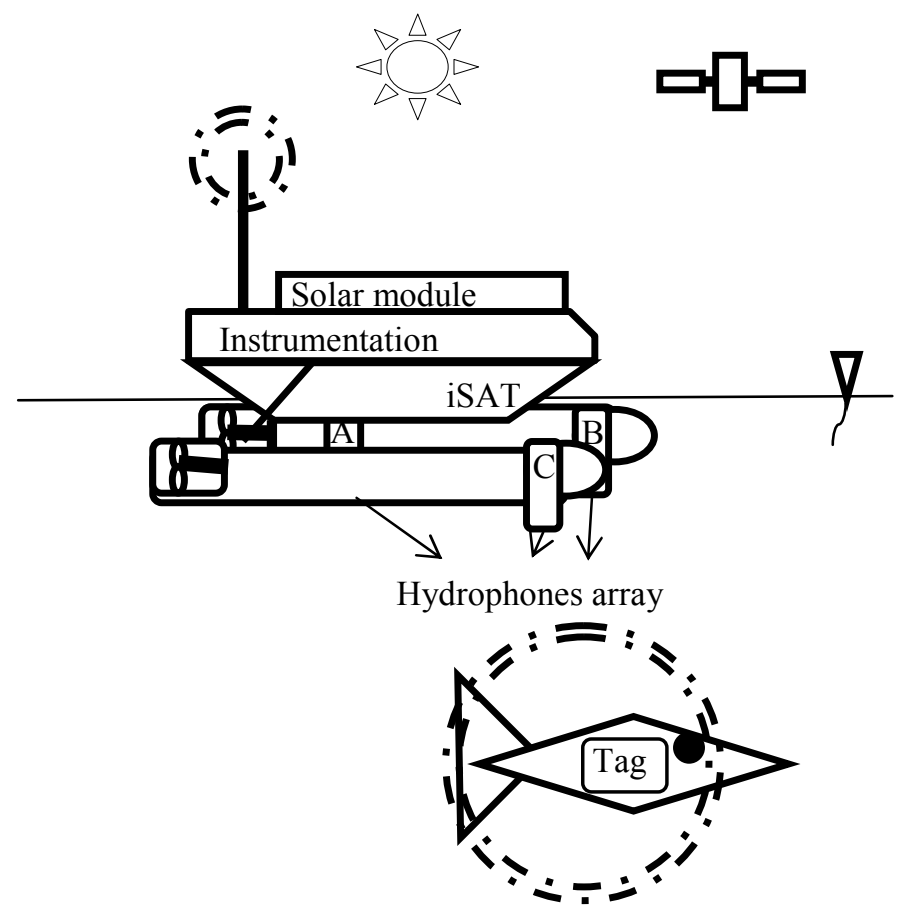

Figure 1 iSAT combines acoustic technology underwater with satellite communications in the air. A B C are the hydrophones of the USBL telemetry system. The fish is tagged and the tag is broadcasting a signal. 
targeted species. The data collected by iSAT describes the movement behavior of marine species with unprecedented detail. Fig 1 is a representation of iSAT following a tagged fish.

\section{ISAT SYSTEM DESCRIPTION}

iSAT is an instrument capable of tracking marine pelagic species autonomously. To achieve this, the system needs to independently manage tasks including navigation, power control, communication, and others. All these tasks are performed by a set of subsystems that together comprise the iSAT. This section describes each of the components that make the satellite-acoustic tracking. However, the research platform is not limited to tracking. Several oceanographic applications are envisioned for the vessel, each requiring specific sensors for the physical variables that need to be measured. For that reason the system is designed to be modular and easily expandable to extend the capabilities of the instrument.

\section{A. The distributed control system}

A master controller board was designed to administer all the tasks on iSAT. Fig. 2 shows the modules hierarchy of the design. This board times operations with a real time clock

Master controller board

RTC, memory, power distribution, I2C
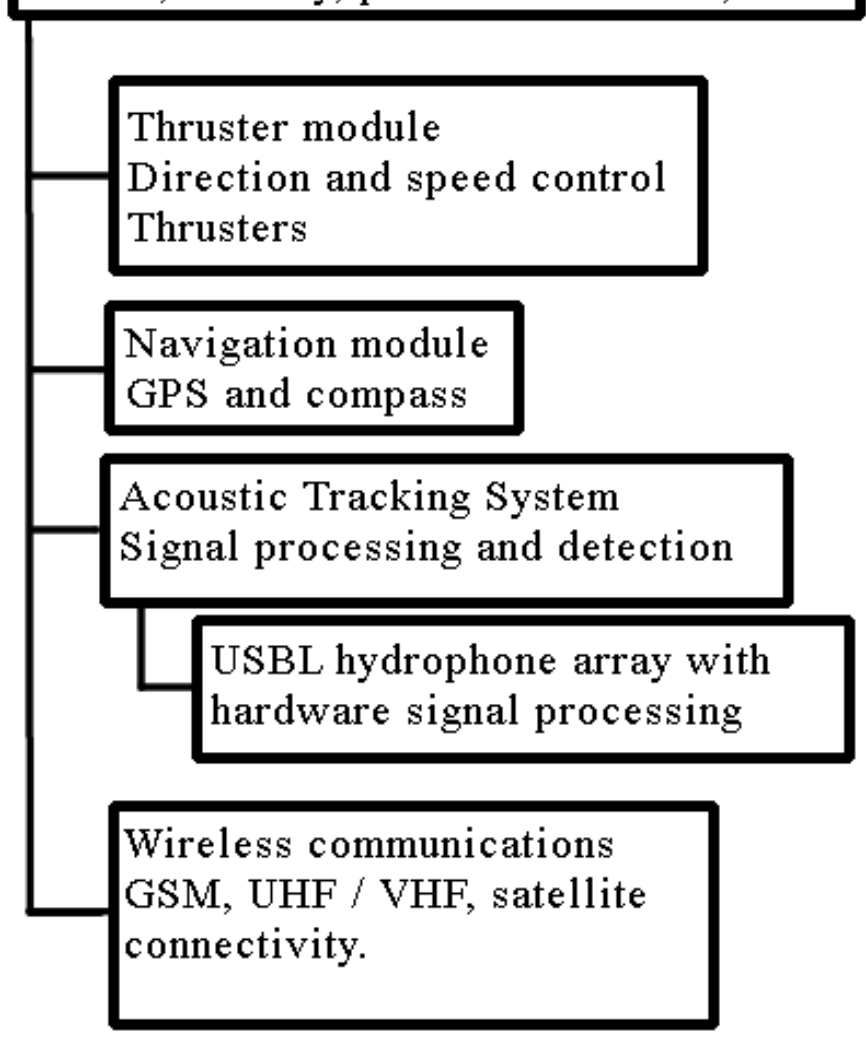

Figure 2 iSAT is a modular system with a master-slave hierarchy of communication that uses the $\mathrm{I}^{2} \mathrm{C}$ protocol. Initially, these four modules govern the operations on board
(RTC) and synchronizes the activities of the other modules. It is also in charge of collecting and storing the information gathered by the different sensors installed on iSAT. It is the only board with a universal serial bus (USB) interface for programming and data download. It manages power by controlling the charge and discharge cycles of the batteries on board using available solar energy for the process and it sends a regulated voltage to the other boards for their processes. The main board, as well as the modules that communicate with it, use the same type of microcontroller in different setups to accomplish their tasks. Communication between modules is performed following the $\mathrm{I}^{2} \mathrm{C}$ protocol, where each module has a different slave address and the messages are sent based on channel request.

\section{B. The propulsion and power system}

The vessel moves with two hi-flow thrusters. They are brushless DC motors located in the aft on the port and starboard sides, behind the pontoons of the swath. Each thruster has a control module that combines a high power stage with a control module regulating direction and speed of rotation. The maximum speed that iSAT can achieve in calm sea conditions is 4 knots, but future adaptations in power and propeller size can make it go faster (albeit with higher power consumption). The energy comes from a set of rechargeable lithium polymer battery bank. The size of the bank is changed so as to offer optimum recharge cycles and minimize wear off the battery capacity. Solar energy is harvested using a polycrystalline photovoltaic solar module, extending iSAT's range by providing a source of renewable energy. This module is installed in the deck and exposed to the sun constantly. The vessel has power autonomy for 1 day under normal sea conditions. It includes an amount of battery power for situations when solar charging is not possible.

\section{Acoustic Tracking System (ATS)}

The ATS consists of two components: an acoustic tag that needs to be inserted into, attached on, or fed to the animal to be tracked and an ultra-short baseline (USBL) array of hydrophones mounted under the vessel's keel. Three hydrophones for tracking are submerged: two in the bow (one starboard and one port) and one straight under the center of gravity of the vessel. The tag has three components: an actuator that generates acoustical waves, a driving circuit, and a battery. The material of the actuator is lead zirconate titanate (PZT), widely known for its piezoelectric properties. The module microcontroller generates a signal with a specifically designed waveform that the actuator transmits acoustically into the water. The pulse is generated at a set frequency with a certain repetition rate that can be adjusted for different marine environments. The power of the acoustic source, at the frequency selected, should enable the signal to travel at least $1 \mathrm{~km}$ in total. On board iSAT, the USBL is made out of two parts: the array of three hydrophones installed on a triangular frame, submerged under the keel, and an acoustics electronic module (fig. 3). A custom hydrophone was built for iSAT. The material of the transducer is the same as that of the actuator (PZT). Preamplifier and amplification stages were designed to condition the target signal for processing. The preamplifier filters low frequencies; i.e. the cut-off frequency is selected so 


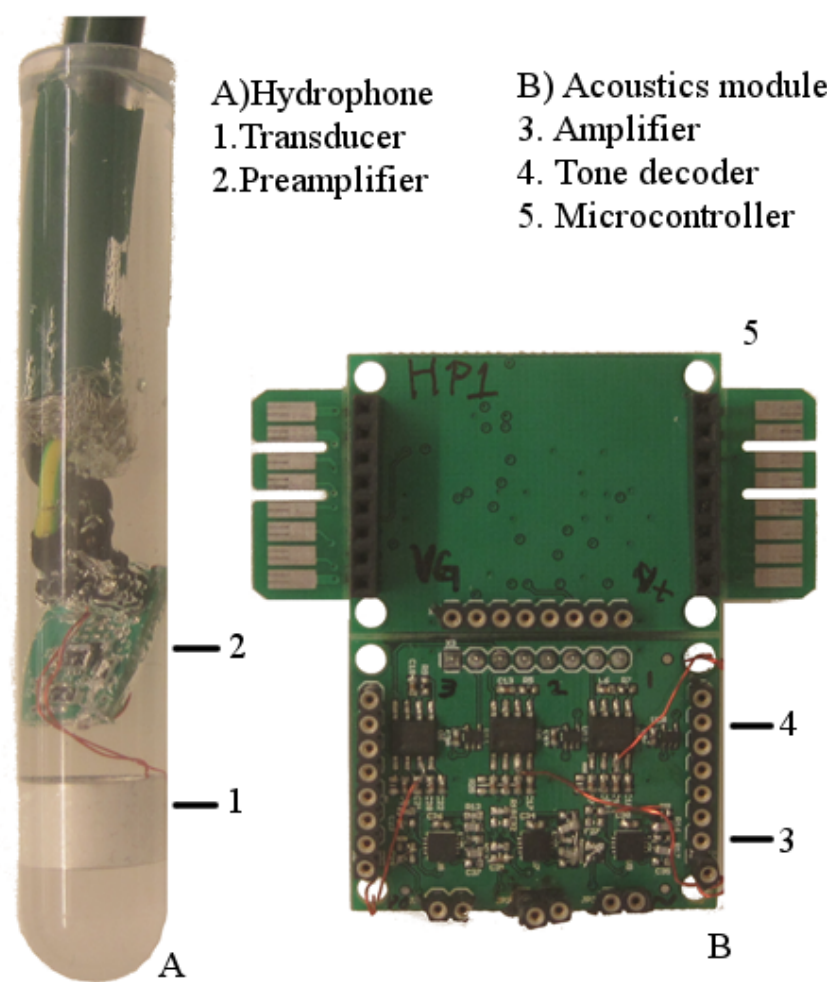

Figure 3 The Acoustic tracking system on board: the hydrophone and the acoustics module receive and process the signal emitted by an acoustic tag attached to the target species.

most of the environmental noise is filtered and amplifies the rest of the spectrum. The transducer and the preamplifier are potted together in a urethane cylindrical hydrophone and submerged directly under the vessel. The acoustic tracking module, on board, amplifies the signal received from the hydrophone and filters it. Once the signal has been extracted, a phase locked loop (PLL) system detects the presence of the signal with desired frequency and generates a pulse that is read by a microcontroller a digital line. There is a signal detector for each of the three hydrophones installed. The ATS has adjusts itself for the changes in speed of sound with respect to the water temperature for more accurate readings. The ATS allows iSAT to stay within $500 \mathrm{~m}$ of the animal, tracking it effectively, but it is programmed to avoid getting closer than $50 \mathrm{~m}$ so as to not alter the species behavior.

\section{The deployment platform}

The electronic circuitry, battery bank, acoustic array and other sensors are installed into a vessel the size of a single person kayak, as it shown in fig. 4. The next generation of iSAT will use a small-waterplane-area twin hull (SWATH). This design was selected for its high stability properties under adverse sea conditions. On the deck, the solar panel and the communication antennas are exposed at all times. All instruments are sealed and waterproofed for protection.

Capsizing is a potential problem for a vessel where most of the time, the wavelength of the sea surf is larger than the length or width of the boat, like in this case. The SWATH mass distribution positions the center of gravity very low, close to the level of the pontoons, so that if the vessel turns over, it will take little effort to turn it back into an operational position.

\section{E. Wireless communication networks}

iSAT has three alternatives on board for communications. For the short range, a mobile network module has been installed. This is limited by the coverage of the local service provider. In Saudi Arabia, mobile range covers to around $5 \mathrm{~km}$ offshore near coastal towns and cities. For short to mid - range communication, and only when there is a clear line of sight between the operations base, which can be a land based center, or a research vessel nearby, and the iSAT vessel, ultra high frequency (UHF) radio is the preferred option to communicate. This is an effective yet inexpensive communications system. Finally, for the long-range communications, the Iridium satellite network is used to transmit data and receive instructions. This network is constantly available, and the data is transmitted to a server that processes text messages and decodes information.

\section{F. Navigation autonomy and range}

The vessel carries on board an electronic compass to determine its heading. A global positioning system (GPS) module provides instant location of the vessel. Both instruments provide feedback to the main board continuously and give it a reference for navigation. To avoid geographical obstacles, like reefs, sand flats, mangrove areas as well as to avoid crossing over political divisions in the Red Sea, a navigation chart is parameterized in terms of GPS coordinates. Exclusion areas are saved into the navigation chart of iSAT so it avoids them. It is expected that while tracking animals, however, this will be a minimal problem since large pelagic species such as whale sharks are unlikely to enter areas not navigable by iSAT.

A set of actions and maneuvers are programmed into the navigation module. They govern the iSAT when it is in a situation different than normal conditions. For example, a "lost tag" program drives the vessel when the tag is out of range or is otherwise not detected. The procedure is to search in a straight line (maintaining the current heading) at full speed for $5 \mathrm{~min}$ before stopping to listen for the tag again. An "emergency" procedure is activated whenever the vessel cannot move from its location, either because it landed or hit a geographical obstacle, or if it is removed from the water while in operation. It transmits its position continuously using any of the

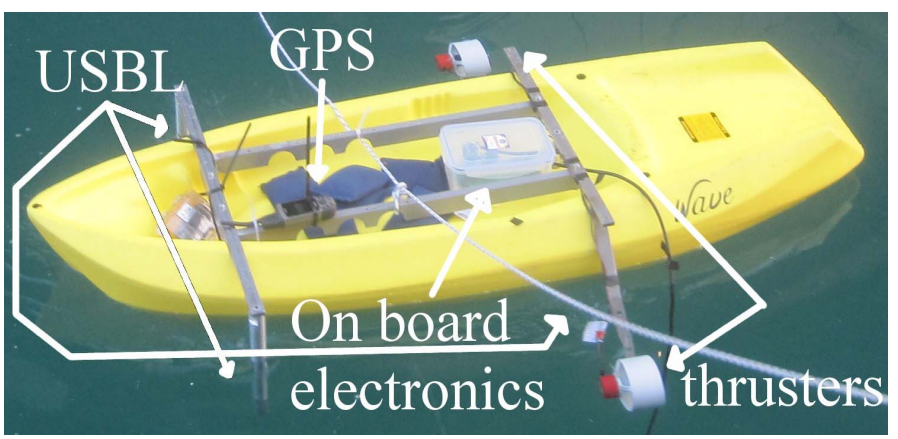

Figure 4 The first generation of iSAT instruments were mounted on a kayak. 
communication methods available until rescued or until the batteries are exhausted. Finally, a "back to base" command sequence returns the vessel to a set 'base' location for maintenance, recovery or to take shelter from adverse conditions.

\section{DIRECTIONALITY AND ACOUSTIC TRACKING}

The tag generates a unique acoustic signal waveform that travels in the water column and reaches each hydrophone in the USBL array at different times. The microcontroller records the order of signal arrival and iSAT determines the relative position of the source within one of the sextants generated by the triangular array, according to the order of arrival of the ping. Continuous correction of the direction keeps the vessel tracking the tagged species. While tracking, the installed sensors gather data that is related to a time stamp generated by the controller board together with its GPS location. In this way, iSAT delivers a set of geographical points with a spatial resolution that can be controlled by changing sampling rate before deployment, or while in action, wirelessly.

The system is designed to monitor the behavior of the whale shark (Rhincodon typus) in the Red Sea. An ongoing study [6] with acoustic telemetry off the coast of Al-Lith, in Saudi Arabia, indicates a seasonal behavior of this species and a localized habitat during the season. Also, the same study indicates that they spend a considerable amount of their time within the first $60 \mathrm{~m}$ of the water column and keep an average speed of 2 knots.

\section{CHALLENGES OF ISAT}

The challenges for successful operation of the iSAT include, but are not limited to: tracking problems, navigation, ineffective communications and others. Although theft and collision have also been considered as potential risks, we prioritize other major challenges above these. The Red Sea is acoustically convenient for the deployment of iSAT, considering its temperature distribution from the nearhomogeneus salinity distribution from the north to the south to the Suez Canal and the Gulf of Aden. Reverberation and Doppler shift effects will act detrimentally on the capacity of iSAT to successfully track a shark. Regarding power administration, sun energy is widely available the Red Sea. Submerging a panel has been considered as an alternative to remove sediments out of the solar screen and improve its performance.

iSAT is constantly undergoing testing. Future activities to be performed include optimization of the range of the current hydrophones, and tracking algorithm. The swath design should be capable of transporting the instruments in the Red Sea safely and to avoid capsizing or be able to go back to surfing position even in the worst weather. Improvements in power administration and maximum energy harvesting will increase iSATs range and reduce time between maintenance operations. For that reason, tests of solar panel performance and battery energy storage are ongoing. Thruster tests will result in the optimum point of use of the available power for a given speed. These experiments increment iSAT capabilities, reduce the risk of failure and make out of it a more useful instrument.

\section{CONCLUSIONS}

The integrated Satellite Acoustic telemetry (iSAT) system is designed and built in KAUST to describe the movements of whale shark (Rhincodon typus) in detail. It is a mobile sensor platform that provides the scientific community with a high resolution description, in the order of 10 s to 100 s of meters, of the paths travelled by marine species and describes the environmental conditions with in-situ measurements of a wide range of marine sensors. The platform is always exposed to the atmosphere allowing positioning information to be retrieved at all times and stored data to be transferred effectively using telecommunication networks such as satellite and mobile to a base for processing. A time stamp is linked to a GPS position of the vessel along with the variables measured by the sensors installed on it. Tracking is performed with a ultra-short baseline acoustic system that processes a signal from a tag embedded into a marine species. iSAT combines the strengths of acoustic telemetry, with the advantages of satellite tracking systems into a single, embedded and autonomous vessel.

\section{ACKNOWLEDGMENTS.}

The authors would like to thank the Coastal and Marine Resources Core Lab for providing the facilities to test the instrument and to outline the help of Ajay Sancheti for his contributions in the development of the project.

\section{REFERENCES}

[1] R. M. Starr, E. Sala, E. Ballesteros, and M. Zabala, "Spatial dynamics of the Nassau grouper Epinephelus striatus in a Caribbean atoll," Marine Ecology Progress Series, vol. 343, no. 2002, pp. 239-249, 2007.

[2] J. Q. Welsh, R. J. Fox, D. M. Webber, and D. R. Bellwood, "Performance of remote acoustic receivers within a coral reef habitat: implications for array design," Coral Reefs, Feb. 2012.

[3] D. Parsons et al., "Snapper Pagrus auratus (Sparidae) home range dynamics: acoustic tagging studies in a marine reserve," Marine Ecology Progress Series, vol. 262, pp. 253-265, 2003.

[4] J. R. Sibert, M. E. Lutcavage, A. Nielsen, R. W. Brill, and S. G. Wilson, "Interannual variation in large-scale movement of Atlantic bluefin tuna (Thunnus thynnus) determined from pop-up satellite archival tags," Canadian Journal of Fisheries and Aquatic Sciences, vol. 63, no. 10, pp. 2154-2166, 2006.

[5] S. G. Wilson, J. J. Polovina, B. S. Stewart, and M. G. Meekan, "Movements of whale sharks (Rhincodon typus) tagged at Ningaloo Reef, Western Australia," Marine Biology, vol. 148, pp. 1157-1166, 2006.

[6] M. L. Berumen, unpublished 